\title{
Imagining the Future of Photoacoustic Mammography
}

\author{
Simone van der Burg
}

Received: 10 March 2008/Accepted: 18 June 2008/Published online: 15 July 2008

(C) The Author(s) 2008

\begin{abstract}
How can a realistic ethical imagination about the future of a technology take shape? This article contains a reflection which is based on the experiences of an embedded ethicist in the context of biophysical research conducive to the development of photoacoustic mammography, which is intended for the non-invasive detection of breast cancer. Imagination in this context already informs the activities of the biophysical researchers, but its role is limited: biophysical future scenarios concentrate on the technological advances that photoacoustics could bring about. In this article it is argued that it is advisable to also consider the medical practice and the ways in which this practice is likely to change as an effect of the introduction of photoacoustic mammography into it. On the basis of this more encompassing imaginative endeavor it is possible to get a clearer idea about how new technologies are able to contribute to human well being, which is informative for the setting of research-goals/priorities and a responsible implementation of new technologies into the world.
\end{abstract}

Keywords Imagination - Embedded ethicist · Breast cancer · Technology research $\cdot$ Human well being

In the Netherlands STW - the most important Dutch public financer of technology research-has started a pilot project of four embedded ethical researches. These are the so called ethical parallel researches, that are carried out parallel to the research of technological researchers. The idea behind this type of research is that ethicists should not pass judgment on new technologies after they are already researched and developed, but during the research process, thus allowing technology researchers to

S. van der Burg $(\bowtie)$

Faculty of Behavioural Science, University of Twente, Postbus 217,

7500 AE Enschede, The Netherlands

e-mail: s.vanderburg@utwente.nl 
actually do something with the criticism and suggestions that they get from ethicists. In this way ethicists who are engaged in the technology research process are thought to be able to co-shape new technologies in ethically sound ways.

It is not yet clear whether it is realistic to expect technologies to change as an effect of the simple engagement of one ethicist in the research phase. There are a lot of obstacles to such cooperative co-shaping; for example, the research phase in which the ethicist is engaged is but one of the episodes in the generation of a new technology, but a lot has been decided before the ethicist came on the scene and a lot will be decided after the ethicist left. Before the research phase the research plan has been written and selected for funding and the researchers accepted the responsibility to realize the goals of that plan, which makes it hard to change those goals; and after the research phase a producer will invest in the further research and development of the technology into a useable product. If ethical reflections that play a role during the research phase are not sustained in these other phases, it is the question whether embedded ethicists exercise any influence at all over the resulting technology.

Another obstacle is that it is quite new to engage an ethicist in the research phase, and this requires changes in the practice of researchers as well as in ethics. Researchers expect ethicists to adopt the role of an outsider-judge who utters judgments with a negative heuristic - that is, which express what not to do, or what harms to avoid-and therefore it is unsurprising that they look at the ethicist with suspicion, for they are afraid she will tell them to stop doing this research. On the other hand, co-shaping a technology for ethicists demands the adoption of a role that is quite unfamiliar to them; namely, the role of an 'insider' - a participant, or rather 'parallel participant'-in the research practice. While some authors-such as Alasdair MacIntyre and Michael Walzer [1-3] — have already defended the view that (ethical) critics should adopt such an insider-position in theoretical accounts, and that they should try to contribute to the improvement of such practices. But they have not reflected on question what form this should take in the specific practice of a technology research.

This article reflects about how ethicists who are embedded in a context of technology research could carry out their task. In order to come to grips with the technology that technological researchers are striving to realize, and that an embedded ethicist is expected to co-shape, it seems wise to think about the question how one could realistically imagine the future of a technology. Such an imagination could help to form an ethical language with a more positive heuristic, which expresses advises about what to do that help to form a future technology in a way that contributes to human well being.

The background of this article is my own work as an embedded ethicist in the context of research at the Institute for Biomedical Technology at the University of Twente (the Netherlands) into a new technology called 'photoacoustic mammography', which is a hybrid technique which uses sound and light for the non-invasive detection of breast tumors. How could an ethicist think about the future of this technology? In this article I would like to imagine the future of photoacoustic mammography, and at the same time do a proposal about how such anticipation can be structured in the context of an embedded ethical research. Such an imagination 
should not be a wild invention; it should be-with a term by Lorraine Code-an 'educated imagination' [4, p. 206]. Just like researchers creatively invent new research-topics in an 'educated' way, building forth on previous knowledge and research methods, ethical imagination should also be the product of a controlled reflection: it should avoid the pitfalls of an ethic that either leaves too little room for imaginations about the future, or departs in highly speculative hypotheses about the future of technologies that have little chance of ever becoming reality. ${ }^{1}$

In this article I propose to start this controlled imaginative endeavor with the future scenarios that technology researchers themselves aim to realize. I call their future goals the 'technical scenarios'. But I will also show that these technical scenarios have limitations; they are primarily concerned with the technological change that a new technology may produce. It seems to me that an ethicist can assist in enlarging the imaginative scope that directs technology research to also include the ways in which a new technology is able to change the medical practice in which it will be used, in better and in worse ways for human well being. This enlarged imaginative endeavor provides material that helps to reflect more responsibly about what research goals to choose, or how to set priorities, and about what to pay attention to during the phase of implementation in the future.

\section{Step 1: Learning About the Technical Future Scenarios}

Photoacoustic mammography builds forth on Near Infrared (NIR) optical imaging technologies that are being researched at different institutes, among others in the US, Great Britain and the Netherlands for their capacity to detect cancer [11-15]. NIR optical imaging images the excessive growth of bloodvessels around tumors the breast, a process termed 'angiogenesis' [16]. NIR optical imaging points out that it is possible to image these vessels in a suspicious area in the body when one points a light beam of near-infrared laser light at it and notes the degree of light-absorption. Higher absorption is taken as a signature of a vascularized tumor [13, 14]. The optical imaging technique is a promising technique because it avoids ionizing radiation, it is relatively inexpensive, and it does not require severe breast compression.

However, the optical imaging technique produces images with poor resolution because the blood vessels it pictures are located in the body and surrounded by tissue which strongly scatters the light. The poor resolution of the image makes it hard to distinguish clearly between the diseased and the healthy part of the body. The photoacoustic technique-also referred to as optoacoustic-is an attempt to

\footnotetext{
${ }^{1}$ A lot has been written about the ways in which imagination of the future can benefit ethical reflection about technology. For e.g. [5-10] But not all ethicists are convinced that imagination should have a role in ethical reflection on technology. While some ethicists think the public debate about technology suffers from a lack of imagination and ethicists should make it more creative [7-9], others argue that ethicists should refrain from imaginative endeavors because they start to discuss cases that are far from realistic [10]. According to Alfred Nordmann ethicists would do better to focus on 'visions that underwrite ongoing research' which include presuppositions in scientific research about, for example, the mechanical nature of human bodies, an idealistic view of autonomy or a consumer interpretation of choice [10, pp. 45-46].
} 
avoid these problems by means of the use of a combination of ultrasound and laserlight, which is invented as a way to distinguish between the absorbed photons and the scattered photons, and concentrates on the absorbed ones for the signal [15]. Several research groups have investigated ways to realize a photoacoustic technology [17, 18]. Researchers of the Biophysical Engineering Group in Twente invented an original way to use sound and light for breast cancer detection, and a first test suggests that it has promising results [19]. The Twente method is to use a pulsed light beam that produces a temporal temperature raise and a short expansion at locations where the light is absorbed by blood, followed by a temperature drop and a contraction $[20,21]$. The thermal expansion resulting from the temperature rise, produces a sound wave that can be pictured with an ultrasound detector.

What are the advantages that photoacoustic mammography could bring about in the future? I did numerous extensive interviews with five researchers; one postdoctoral researcher who carried out the research, two members of the permanent scientific staff, a full professor and an assistant professor, and a radiologist and an oncologist who took part in the testing on patients. In those talks I found out that several future scenarios figure in the minds of the researchers, which I articulated, distinguished, ordered and discussed with them. Each scenario explores different possible future capacities, to which the researchers refer with the terms 'specific' and 'sensitive'. Sensitive technologies have screening potential; they are able to detect a lump, but they have limited capacity to distinguish between a malignant and a benign lump. ${ }^{2}$ Specific technologies, by contrast, are able to determine the diagnosis; they specify which lumps in the breast are harmless cysts and which ones are cancer. In the present medical practice there is a distinction between the technologies that are used for screening and the technologies that are used for diagnosis. Most often ultrasound and X-ray mammograms are made to screen whether there is a lump there; a biopsy determines whether the lump is cancer or not. Generally X-ray mammography is thought to set the 'golden standard' for screening and the biopsy for diagnosis, which means that the capacities of all newly invented instruments for screening and diagnosis are evaluated respectively in comparison to the performance of X-ray mammography and biopsies.

In the future scenarios of photoacoustic mammography, the researchers imagine their technology to offer an alternative to the technologies that are accepted at present as the 'golden standard'. While they are modest about what they are able to deliver now, and they continually affirm that a lot further research is needed before any of the capacities of the imaginary future photoacoustic mammography can be realized, the following scenarios do figure in their thoughts about what their research-activities are eventually aiming at. In the first future scenario, they ascribe to photoacoustic mammography primarily a diagnostic role. In this scenario photoacoustic mammography would replace part of the biopsies. It would thus be primarily a diagnostic tool, a specific technology, which would be used after a lump has been detected with an X-ray mammogram.

\footnotetext{
${ }^{2}$ For example, a sharp edge of the lump in the breast, as opposed to a diffuse one, is in most cases-but not all-a sign that it is a benign anomaly.
} 
The second scenario further explores the potential of photoacoustics as a specific technology. The aim of this research is to find out whether photoacoustics is able to offer information about the amount of oxygen in the blood surrounding a tumor, because blood with a lot of oxygen absorbs less light than blood with little oxygen, which has a darker color. Oxygenation of the blood is thought to be indicative of the speed with which the tumor is growing: little oxygen is expected to mean that the tumor grows fast, for it needs oxygen to grow. If photoacoustics is able to offer information about oxygenation, the technologists think it may be able to offer information that is important for the physician's choice for adequate treatment. However, this research is not the primary interest of the radiologist and the oncologist who are engaged in the testing of photoacoustic mammography on patients, because there are no treatments available at present that aim specifically at fast or slow growing tumors.

The third and the fourth scenario focus also on the sensitive potential of the technology. In the third scenario photoacoustic mammography would not only be used for diagnosis, but also for screening. It would thus have specific as well as sensitive capacities, and could eventually replace the biopsies and the X-ray mammography. This is the scenario that interests the oncologist and the radiologist most. In fact they talk about this technology as if this is the only future that it is intended to bring about. The scientific staff agrees that this scenario is important and valuable, and that it is possible that this will become reality. But some are a little bit more skeptical than others whether it is feasible. Whether photoacoustics will become successful as a screening technology depends on whether this technology will be able to detect cancer in an earlier phase than X-ray. That means that further research should establish whether or not the formation of extra vessels which can be detected with photoacoustics can be seen before a lump is formed that can be detected with X-ray.

The fourth scenario comes forwards in the expression of the researchers' interest to investigate the possibilities of this technology to diagnose cancer in glandular breasts. Glandular breasts are hard to picture with X-ray mammography; a lot of pictures have to be taken in order to get a clear view of what is inside, which is harmful with X-ray's ionizing radiation. And even when the breast is pictured from all sides, X-ray mammograms miss up to $25 \%$ of the breast cancer in glandular breasts [22]. This is known to be a problem especially for younger women, for it is known that breasts become less glandular with age. While it is expected that photoacoustic mammography will also have to take a lot of pictures in order to traverse breasts with a lot of glands, it is thought to be able to offer an accurate image and it uses harmless radiation. This capacity of the technology is not currently being researched, but it is a very important research-topic that the researchers want to explore in the near future.

An overview of these scenarios show that the researcher's imagination about the future capacities of photoacoustics focuses primarily on technological advances: the researchers look at the technologies that are used in the present medical practice and look how photoacoustics is able to transcend the limitations of these technologies. In comparison to a biopsy, a noninvasive photoacoustic image offers a painless way to diagnose. And it may produce information on the speed of growth that is 
unavailable at present. In comparison to X-ray mammography, a photoacoustic mammogram uses harmless radiation, the breast does not need to be pressed that hard to get the picture (which reduces pain) and it may be able to produce better results on young women. In comparison to NIR optical imaging, photoacoustics offers an image with a better resolution.

In comparison to all these technologies, photoacoustic mammography is thought to be able to offer an improvement. What the researchers do not take into account in their future imagination, is how the introduction of photoacoustics in the medical context may change that context, and accordingly the lives of people. However, when they exclude the medical practice in their reflections, they do not expand their imagination in ways that are ethically informative. That is what I would like to show in the next two paragraphs.

\section{Step 2: Learning About the Practice to Which the Technology Contributes}

The medical practice for which photoacoustic mammography is intended is the practice of breast cancer screening and diagnosis. This can have many forms. It could for example mean that women who feel an anomaly in one of their breasts are sent by the general practitioner to a hospital where they would have a photoacoustic mammogram taken, in stead of an X-ray mammogram or an ultrasound image, sometimes with a biopsy, which is the practice at present. But the most important reason why this technology is economically interesting is that it could be used in the preventive public screening service, which has been gradually implemented in many European countries and in North America during the past decades. This preventive screening service is most commonly offered annually or bi-annually to a population of women past the menopause, between 50 and 70 or 75 years of age. Especially the use in this screening practice needs attention, for since 2000 there has been a fierce discussion over the utility of these preventive screening services with X-ray mammography which remains inconclusive until this day. The disagreement concentrates on two issues, namely (1) that it remains unclear whether screening with X-ray mammography reduces breast cancer death rates (and with what number they are reduced) and (2) that the unavailability of knowledge about the reduction of the death rates makes it impossible to determine whether the benefits of screenings outweigh the costs in terms of physical harm due to the ionizing radiation (the ionizing radiation of $\mathrm{X}$-ray is expected to have a carcinogenic potential) or to unnecessary treatments (following on false positive results), inconvenience, emotional stress and physical pain because of the compression of the breast.

What are the reasons to doubt whether screenings are beneficial? During the 1980s and 1990s many studies have been done in North America and in different European countries that pointed out that frequent screenings-like, say, annually or biannually - help to reduce breast cancer mortality. But not all studies produced this favorable result. One famous large scale Canadian study did not find a reduction of breast cancer death rates as a result of the introduction of annual screenings with X-ray mammography [23-26]. And a smaller study in the Swedish county Malmö also did not show a benefit of screenings. Both studies, especially the Canadian one, 
were severely criticized. However, in 2000, two Danish researchers, Peter Gotzsche and Ole Olsen, working at the well respected Nordic Cochrane Center in Copenhagen published a critical survey in the Lancet of all scientific research about the relation between screenings with X-ray and the reduction of breast cancer death rates, and they argued that all but two of the trials were flawed in design to the effect that the results were unreliable [27]. In their opinion the Canadian study and the Malmö-study were most reliable.

The Danish researchers came to this conclusion on the basis of a critical survey of the way in which eight extensive trials were carried out; beside the Canadian and the Malmö study they judged all of them to be biased. They remarked for example that a lot of these trials excluded women who had been diagnosed with breast cancer previous to the trial, which influences the final results; for women who have had breast cancer in the past are more likely to eventually die of breast cancer than other women, and when they are excluded the number of deaths in the screened population is likely to drop. Next to that, they criticized some trials because they worked with unbalanced test and control groups. In a reliable trial the test group and control group are exactly the same. However, in trials that were carried out in New York and Sweden the number of women in each group was not the same, and even remained unclear. ${ }^{3}$ When the number of people in the screened study group is smaller than in the control group (which was the case in New York) this introduces a bias in the study in favor of the screening group. Also, many studies did not verify whether the study group and the control group consisted of similar populations; in the New York study there were imbalances in both groups about the medical history (previous lumps in the breast), age (pre or post menopausal) and education level. In the Edinburgh study $26 \%$ of the control group was in the highest socioeconomic stratum, compared with $53 \%$ in the screening group. In Stockholm and Götenborg the study women were on average younger than the women in the control group. Additionally the Swedish trials contained another mystery: while for every 1,000 women screened biannually for 12 years, one breast cancer death is avoided, the total number of deaths increased by six [28].

All of the trials that Gotzsche and Olsen condemned biased pointed out that screenings with X-ray mammography help to reduce breast cancer mortality. The only two trials they judged to be unbiased pointed out that screening for breast cancer with mammography had no effect on breast cancer mortality. From these findings the researchers "(...) conclude that screening for breast cancer with mammography is unjustified." [27, p. 133]

Next to the opinion that breast cancer screenings do not reduce the amount of breast cancer deaths, Gotzsche and Olsen also argue that screenings inflict harm, because they lead to over diagnosis and over treatment. Both of the unbiased trials show that surgery and radiotherapy is significantly more common in groups of women who are screened than in women who are not screened for breast cancer.

\footnotetext{
3 In the New York trial the number varies between 31,092 and 30,000 in the study group, and between 30,565 and 30,765 in the control group [27, p. 129]. In the Stockholm trial the number of controls is 20,000 in an early report and 19,943 in the final report. In the final report of the Swedish trials the number of women in the screened group fell from 40,318 to 38,525 and increased in the control group from 19,943 to 20,651 [27, p. 130].
} 
This seems natural, for when there is screening, more tumors are found, so more treatment is needed. However, the Danes think it is also possible that the number of treatments will augment because of "(..) false-positive findings, cell changes that may never develop into cancer, and cancers that will develop so slowly that the woman dies of other causes before the cancer becomes apparent." [27, p. 133] ${ }^{4}$

This observation by Olsen and Gotzsche was the start sign for a fierce debate. Critics of this Danish view noted that the flaws that Olsen and Gotzsche identified were not severe enough to discount the body of evidence that supported the view that screenings reduced the death cancer death rate [29, 30]. Even if the trials were not securely carried out, and they do not offer exact information as to how many breast cancer deaths are prevented with screening, they could not be completely discarded either. The Danes, however, continued to reassess their findings [28, 31]. For example, in an article in 2001 in which they report also new evidence which shows that screening brings about an increase in the number of surgeries, chemotherapy and radiotherapy. In this article this is reason for them to suspect that screening will not reduce, but increase overall mortality because of cardiovascular effects [28].

In the years to follow the debate flared up each time a new trial was presented, but the disagreeing parties never came to an agreement. ${ }^{5}$ Eventually the parties just became tired of the debate and stopped repeating their standpoints [22]. The only randomized study that appeared and which is sensitive to the criticism, while also showing that screening has a significant but modest effect on mortality from breast cancer, is a Swedisch study by Nyström and his colleagues from 2002. This study is currently cited as the only trustworthy one that shows a positive effect of breast cancer screenings with X-ray mammography on breast cancer death rates. The Nyström-study shows that reduction in breast cancer mortality is 27\% among 55-64 year old women, and deaths were reduced $14 \%$ among those forty-five to fifty-four who were screened [35]. But until now this has been the only reliable study that shows such a favorable effect. The Canadian study and the one carried out in Malmö do not show it.

\footnotetext{
${ }^{4}$ The Canadian trial especially fueled this argument against screening, for it pointed out that the number of benign findings in biopsy samples was two to four times higher in the screened groups than in the control groups.

${ }^{5}$ For example, at the presentation of another Swedish research, which used the data of the regional oncology Centers in Uppsala and Linoping in Sweden to compare deaths from breast cancer in the twenty years before screening was introduced (1958-1977) with those from breast cancer diagnosed in the twenty years after the introduction of the screening (1978-1997). The findings show that among women aged forty to sixty-nine who were screened, there was lower breast cancer mortality to those diagnosed prescreening. In the twenty to thirty nine age group, there was no significant difference in breast cancer mortality in 1978 to 1997, compared with 1958 to 1977 [32]. But Peter Gotzsche wrote in a short response that this historical approach is also unreliable [33]. He argues that screening predominantly identifies slow-growing tumors, so women with breast cancer diagnosed during screening generally have a better prognosis, than the women diagnosed pre-screening for these diagnoses also included the quick growing tumors, which are more dangerous. According to Gotzsche a randomized study is needed to determine the effect of screening on death rates, but of course he criticized already all randomized studies because of the unbalanced test and control groups. This criticism also affects another research carried out in the Netherlands [34].
} 
Reading about the inconclusive controversy over the benefits of beast cancer screenings with X-ray mammography, raises questions that are relevant to researchers who are in the process of researching a new technology for that context. A new technology such as photoacoustics could improve the benefits of a screening practice, in the sense that some of the future scenarios that the technology researchers sketch-if they turn out to be realizable-could succeeds to reduce breast cancer death rates as well as the false positive and false negative diagnoses, which would of course help to promote human well being. If it would do that, important arguments against screening which are put forwards in this debate would loose their force.

However, at present the debate over screening with X-ray mammography is generally ignored in the context of research into photoacoustics. While some of the technological researchers who work on photoacoustics took notice of the debate, it did not affect their work. The reason for that is that they consider it their task to study the drawbacks of the technologies being used and try to transcend those in a new technology; they do not think it is their responsibility to think about how to contribute to the improvement of the practice of breast cancer screening so that it helps to improve human well being.

In my opinion this is a missed opportunity. When we reconsider the technological future scenarios that I distinguished in section one and engage in an additional imaginative endeavor to establish whether or not each of these scenarios could contribute to the improvement of the results of the screening practice, we come to another view of what scenarios are worth to try to realize, and which ones present less valuable goals to pursue. That is what I want to show in the next section.

\section{Step 3: Ethical Future Scenarios of Photoacoustic Mammography}

The most important arguments against screening with X-ray mammography that are put forwards in the debate are (1) that screening with X-ray mammography does not reduce breast cancer death rates, or reduces them only minimally and (2) that screenings actually harm women; they cause inconvenience, emotional stress and physical pain to all women but especially to women who receive false positive results and who are offered treatment that they don't need. And next to that, the ionizing radiation of X-ray is expected to have a carcinogenic potential.

What effects would the realization of the technological future scenarios that the researchers sketch have on these drawbacks of the screening practice? According to the first technical scenario photoacoustic mammography would succeed to replace part of the biopsies. That would mean that women with a suspect lump in their breast do not always have to have a painful puncture taken and they can have their results very quickly by means of a non-invasive photoacoustic mammography. In this scenario photoacoustic mammography helps to reduce the physical pain and emotional stress that a biopsy causes. So, photoacoustics in this scenario takes away some of the harm that the current diagnostic practice inflicts, but it does not make it more successful in reaching its goal of reducing the amount of breast cancer deaths. 
The second technological scenario suggests that photoacoustics may in the future be able to point out the oxygenation of the blood around a tumor, and that oxygenation could be informative of the speed with which a tumor grows or spreads. Would this improve the present practice of breast cancer screening and diagnosis? The physician in the research team reports that there is no special treatment for fast and slow-growing cancers available at this moment, so this scenario cannot be expected to lead to a reduction of death rates in any near future.

If the third technological scenario would be realized in which photoacoustic mammography would prove capable of sensitive screening, the technology would could contain more favorable promises for human well being. Next to the expectation that the process of breast cancer screening and diagnosis becomes less burdensome if photoacoustics were used for diagnosis as well as screening-it would shorten the sequence of medical checks and the process would involve less physical pain-using photoacoustics for screening could also help to reduce death rates for it does not use harmful radiation that has the potential to produce cancer, such as X-ray mammography is thought to do. Besides, if photoacoustic mammograms turn out to be able to detect cancer earlier than X-ray mammograms, than it could be possible that screening with photoacoustics helps to reduce the amount of breast cancer deaths in the future.

What about the scenario in which photoacoustic mammography turns out to be able to detect breast cancer in glandular breasts? While this could of course be very beneficial for the well being of young women, who are known to have more glands in their breasts [36], especially to those with a genetic predisposition to get breast cancer. But a probable side-effect could be that it will become very difficult to avoid an extensive enlargement of the present population to be screened. Until now, women younger than 50 years of age have generally been excluded from the screened population in countries that have a public screening service, such as the Netherlands or Sweden. For women beneath 35 years of age the reason for this exclusion is biological: breast cancer is rare among women of that age group. But women between 40 and 50 years of age have a higher chance of getting cancer. This group is generally excluded from the screened population for a technological reason: X-ray mammograms miss up to $25 \%$ of breast cancer in women before their menopause (as opposed to $10 \%$ for women in their fifties and older) [22].

If photoacoustic mammography turns out to be able to make mammograms of pre-menopausal glandular breasts, this is a reason to extend the age-group to be screened. When there is no longer a technological reason to exclude women in their forties, fairness requires to offer screenings to them too. Interestingly, this means that if the possibility to use photoacoustic mammography is realized, it not only opens the possibility to choose to include younger women; it also makes it difficult to avoid including them: it becomes unjust not to do it. ${ }^{6}$

What would the effect of this scenario be on human well being? Of course, if photoacoustic mammography is able to detect cancer in an early phase in young

\footnotetext{
${ }^{6}$ Furthermore, research of the kind of cancers that women in their forties develop may point out that this group ought to be screened more often-for example, annually in stead of biannually which is currently the standard in the Netherlands-because the cancers they develop grow faster. A more frequent screening is of course also more expensive.
} 
women's breasts, this causes an improvement of the well being of young women who develop cancer. But this positive effect has a negative counterpart. An enlargement of the present breast cancer screening practice also means that a larger population of mostly healthy women is subjected to bi-annual or annual screenings. This means an augmentation of the costs of this service and this money can no longer be spent on other important goals, such as reducing the waiting list for surgery or care for the terminally ill.

Next to that, it also seems important to note that an enlargement of the present screening practice may also bring about cultural changes, for example in the experience of health and disease; being healthy might loose its sorrowless nature when one is expected to regularly subject oneself to medical checks. But people may also start to attach much more value to health as a value in their lives, once they're expected to engage in more and more activities to preserve it and therewith loose other important sources of value out of scope. Lastly the extend to which one is held responsible for one's own health (and blamable for loosing it) may change radically, and consequently the norms may change too about who deserves priority on the waiting list for surgery, or who deserves to be insured. ${ }^{7}$

\section{Conclusion}

While the biophysicists who research photoacoustic mammography have a very lively imagination, it focuses narrowly on technological devices and how they can be improved. Their imaginative endeavor does not include the context, the practice, in which the technology will be used. I think an embedded ethicist who is able to broaden that imagination to include also the practice for which the technology is intended, may help to enhance the creativity of researchers and in this way contribute to choices that are well argued for about what research-goals to pursue or to give priority.

At present, the research-goals are primarily determined by the topics that the researchers find most scientifically challenging, as well as the ones they succeed to get funding for. But next to scientific interest, all researchers also claim that they find it important to contribute to human well being. An imagination that includes information on the medical practice for which their technology is intended, and seeks to determine which technological scenarios will enable that practice to promote human well being, can help to make well informed research choices. In the case of photoacoustic mammography this imaginative endeavor has produced the insight that the scenario in which photoacoustics is used for screening is more helpful if one wants to remove the drawbacks of the current practice of breast cancer screening and diagnosis, and help it to become more effective in achieving the goal of a reduction in the breast cancer death. That would mean that an ethicist who wants to promote human well being would advise the researchers to give priority to

\footnotetext{
7 These and similar issues are well described in the extensive literature on ethical aspects of medicalization. Marcel Verweij gave a helpful overview in his article 'Medicalization as a moral problem for preventive medicine' [37].
} 
research that determines whether photoacoustics is a sensitive technology which can be used for screening, and not try to develop its specific capacities for diagnosis further. At this moment however, the primary focus of the researchers is on the specificity of the instrument, not on its sensitivity.

In addition, the broader ethical imagination gives reason to be cautious in a later phase, when the technology will be implemented in medical practice. As was discussed above, the screening practice may enlarge, if the technology turns out to be able to screen cancer in young women's breasts. This is a situation that needs to be anticipated. Careful reflection is needed about whether or not this is desirable, and about how to keep the practice small, if there are other more important medical services that need the budget.

A broadening of the imaginative endeavor thus helps to set research-goals and priorities, and to direct research activities as well as the final implementation of a technology. But it also produces a change in the research practice as a whole. Until now the biophysical researchers understood their responsibility primarily in technological terms; they see it as their task to transcend the limits of the present technologies with new technologies. However, taking the recommendations of an ethicist seriously, who engages in a broader imaginary journey that includes also the medical practice, would imply a reorientation in the reflections about one's own responsibility as a technology researcher. Including an ethicist in such a context, and taking the suggestions seriously that he or she offers, could mean that technology researchers are motivated not only to think about technological innovations they may be able to deliver, but also to take into consideration the effect of those innovations on human (social) life.

Changing the research practice in this way also offers an important subject for discussion with public institutions that offer funding to technological researchproposals. If public funding institutions are interested in the impact of new technologies on human lives, they should make more room for such an evaluation in their assessment of research projects. In that way they could sustain this type of reflection, and enforce its influence in the research practice. At this moment, public institutions in the Netherlands-such as STW or Senter Novem-but also in the US-NSF-express an interest in the 'societal relevance' or the 'broader impacts' of technology research, but it remains rather vague what these terms mean. In my opinion it could mean that the impact of new technologies on human life and practices is anticipated already in an early phase of the research. A broader ethical imagination, as is offered in this article, could help to come to such an anticipation. If funding institutions would enforce this kind of ethical imagination about technology, they could contribute to the development of a reflective research practice that takes seriously the way in which technology changes human life.

Acknowledgements I would like to thank the technological researchers of the Biophysical Engineering Group at the University of Twente for their generous and open cooperation with this research. Also, this article has profited a lot from comments on earlier versions of it by the anonymous referees, as well as my good colleagues Tsjalling Swierstra, Annemarie Mol, Marianne Boenink and Govert Valkenburg. Finally I would like to thank STW for funding the ethical parallel research that resulted in this paper. 
Open Access This article is distributed under the terms of the Creative Commons Attribution Noncommercial License which permits any noncommercial use, distribution, and reproduction in any medium, provided the original author(s) and source are credited.

\section{References}

1. MacIntyre, A. (1981). After virtue: A study in moral theory. Notre Dame, IN: University of Notre Dame Press.

2. Walzer, M. (1987). Interpretation and social criticism. Cambridge, MA: Harvard University Press.

3. Walzer, M. (2002). The company of critics: Social criticism and political commitment in the twentieth century. New York: Basic Books.

4. Code, L. (2006). Ecological thinking: The politics of epistemic location. Oxford University Press: Oxford.

5. Coeckelbergh, M. (2007). Imagination and principles: an essay on the role of imagination in moral reasoning. New York: Palgrave Macmillan.

6. Coeckelbergh, M., \& Mesman, J. (2007). With hope and imagination: Imaginative moral decisionmaking in neonatal intensive care units. Ethical Theory and Moral Practice, 10, 3-21. doi:10.1007/ s10677-006-9046-2.

7. Swierstra, T., \& Rip, A. (2007). Nano-ethics as NEST-ethics: Patterns of moral argumentation about new and emerging science and technology. NanoEthics, 1, 1. doi:10.1007/s11569-007-0005-8.

8. Stemerding, D., \& Swierstra, T. (2006). How might scenariostudies help us to think about the normative implications of genomics and predictive medicine? In A. de Bouvet, P. Boitte, \& G. Aiguier (Eds.), Questions éthiques en médicine prédictive (pp. 81-88). Paris: John Libbey Eurotext.

9. Dijck, J. van, (1997). Het verbeeldingstekort: Over kloning, media, wetenschap en sciencefiction. $K$ \& M: Tijdschrift voor Empirische Filosofie, 21(2), 83-96.

10. Nordmann, A. (2007). If and then: A critique of speculative nanoethics. NanoEthics, 1, 36-46.

11. Franceschini, M. A., Moesta, K. T., Fantini, S., Gaida, G., Gratton, E., Jess, H., et al. (1997). Frequency-domain techniques enhance optical mammography: Initial clinical results. Proceedings of the National Academy of Sciences of the United States of America, 94, 6468-6473. doi:10.1073/ pnas.94.12.6468.

12. Grosenick, D., Wabnitz, H., Rinneberg, H. H., Moesta, K. T., \& Schlag, P. M. (1999). Development of a time domain optical mammography and first in vivo applications. Applied Optics, 38, 29272943. doi:10.1364/AO.38.002927.

13. Tromberg, B. J., Shah, N., Lanning, R., Cerussi, A., Esponoza, J., Pham, T., et al. (2000). Noninvasive in vivo characterization of breast tumors using photon migration spectroscopy. Neoplasia (New York), 2, 26-40. doi:10.1038/sj.neo.7900082.

14. Pogue, B. W., Poplack, S. P., McBride, T. O., Wells, W. A., Osterman, K. S., Osterberg, U. L., et al. (2001). Quantitative hemoglobin tomography with diffuse Near-Infrared Spectroscopy: Pilot results in the breast. Radiology, 218, 261-266.

15. Xu, M., \& Wang, L. V. (2006). Photoacoustic imaging in biomedicine. The Review of Scientific Instruments, 77, 261-266.

16. Carmeliet, P., \& Jain, R. K. (2000). Angiogenesis in cancer and other diseases. Nature, 407, $247-257$. doi:10.1038/35025220.

17. Oraevsky, A. A., Savateeva, E. V., Solomatin, S. V., Karabutov, A. A., Andreev, V. G., Gatalica, Z., et al. (2002). Optoacoustic imaging of blood for visualization and diagnostics of breast cancer. Proceedings of the Society for Photo-Instrumentation Engineers, 4618, 81-94. doi:10.1117/12. 469851.

18. Khamapirad, T., Henrichs, P. M., Mehta, K., Miller, T. G., Yee, A. T., \& Oraevsky, A. A. (2005). Diagnostic imaging of breast cancer with LOIS: clinical feasibility’. Proceedings SPEI, 5697, 35-44. doi:10.1117/12.596916.

19. Manohar, S., Vaartjes, S. E., van Hespen, J. C. G., Klaase, J. M., van den Engh, F. M., Steenbergen, W., et al. (2007). Initial results of in vivo non-invasive cancer imaging in the human breast using near infrared photoacoustics. Optics Express, 15, 19. doi:10.1364/OE.15.012277.

20. Manohar, S., Kharine, A., van Hespen, J. C. G., Steenbergen, W., \& van Leeuwen, T. G. (2005). The Twente photoacoustic mammoscope: System overview and performance. Physics in Medicine and Biology, 50, 2543-2557. doi:10.1088/0031-9155/50/11/007. 
21. Manohar, S., Kharine, A., van Hespen, J. C. G., Steenbergen, W., \& van Leeuwen, T. G. (2004). Photoacoustic mammography laboratory prototype: Imaging of breast tissue phantoms. Journal of Biomedical Optics, 9, 1172-1181. doi:10.1117/1.1803548.

22. Finkel, M. L. (2005). Understanding the mammography controversy: Science, politics and breast cancer screening. Westport, CT/London: Praeger.

23. Miller, A. B., Howe, G. R., \& Wall, C. (1981). The national study of breast cancer screening protocol for a Canadian radomized controlled trial of screening for breast cancer in women. Clinical and Investigative Medicine. Medecine Clinique et Experimentale, 4, 227-258.

24. Baines, C. J. (1994). The Canadian national breast screening study: A perspective on criticisms. Annals of Internal Medicine, 120, 326-334.

25. Miller, A. B., To, T., Baines, C. J., \& Wall, C. (1997). The Canadian national breast screening study: Update on breast cancer mortality. Journal of the National Cancer Institute Monographs, 22, 37-41.

26. Miller, A. B., To, T., Baines, C. J., et al. (2002). The Canadian National Breast Screening Study-1: Breast cancer mortality after 11 to 16 years of follow up: a randomized screening trial of mammography in women age 40 to 49 years. Annals of Internal Medicine, 137, 305-312.

27. Olsen, O., \& Gotzsche, P. C. (2000). Is screening for breast cancer with mammography justifiable? Lancet, 355, 129-134.

28. Olsen, O., \& Gotzsche, P. C. (2001). Cochrane review on screening for breast cancer with mammography. Lancet, 358, 1340-1342. doi:10.1016/S0140-6736(01)06449-2.

29. Wald, N. (2000). Populist in stead of professional. Journal of Medical Science, 7, 1.

30. Duffy, S. W. (2001). Interpretation of the breast screening trials: A commentary on the recent paper by Gotzsche and Olsen. The Breast, 10, 209-212. doi:10.1054/brst.2000.0238.

31. Gotzsche, P. C., \& Olsen, O. (2000). Screening mammography re-evaluated. Lancet, 355, 752. doi: 10.1016/S0140-6736(05)72161-9.

32. Tabar, L., Yen, M. F., Vitak, B., et al. (2003). Mammography service screening and mortality in breast cancer patients: 20 year follow up before and after introduction of screening. Lancet, 361, 1405-1410. doi:10.1016/S0140-6736(03)13143-1.

33. Gotzsche, P. C. (2003). Mammography service screening and mortality. Lancet, 362 , 329-330. doi: 10.1016/S0140-6736(03)13982-7.

34. Otto, S. J., Fracheboud, J., Looman, C. W. N., Broeders, M. J. M., et al. (2003). Initiation of population-based mammography screening in Dutch municipalities and effect on breast cancer mortality: A systematic review. Lancet, 361, 1411-1417. doi:10.1016/S0140-6736(03)13132-7

35. Nyström, L., Andersson, I., Bjurstam, N., et al. (2002). Long term effects of mammography screening: Updated overview of the Swedish randomized trials. Lancet, 359, 909-919. doi: 10.1016/S0140-6736(02)08020-0.

36. Beckett, J. C., Jr., \& Kotre, J. C. (2000). Dosimetric implications of age related glandular changes in screening mammography. Physics in Medicine and Biology, 45(3), 801-814. doi:10.1088/00319155/45/3/316.

37. Verweij, M. (1999). Medicalization as a moral problem for preventive medicine. Bioethics, 13(2), 89-113. doi:10.1111/1467-8519.00135. 